\title{
The political-economy of conflicts over wealth: why don't the rabble expropriate the rich?
}

\begin{abstract}
The most striking feature of liberal democracies is the coexistence of large inequalities of wealth with a roughly egalitarian distribution of voting power. So far most attempts to explain this have asked 'why don't the poor form a coalition to expropriate the rich?' This paper argues that this is not necessarily the best way to interpret the problem and attempts to provide an alternative unified political-economic model that is more consistent with standard assumptions about voting. This is done by studying what would happen if every possible coalition could form in a wealth distribution game. Among the main findings is that, if the marginal contribution of every individual to production is increasing sufficiently, there is a stable distribution of the product. This may include the egalitarian distribution. If individuals are not so valuable there is no stable distribution.
\end{abstract}

\section{Introduction}

Societies that can loosely be called liberal-democratic are characterized by a collective decision making mechanism in which voting power is roughly equally distributed and a distribution of wealth which is strikingly inegalitarian. The Survey of Consumer Finances sponsored by the Federal Reserve Board in the US gives some rough idea of the sort of distribution we might wish to consider. It shows that the top five percent hold about as much wealth as the bottom ninety-five percent and figures seem to be similar for Australia and many EU countries. This gives rise to a fairly obvious question. What would be the distribution of wealth if this were the only, or main, issue and every agent voted in their own interest? This paper considers some aspects of this question.

The argument of the paper is that much of the debate about distribution in political-economy has assumed a natural coalition structure of rich and poor and that this may not be the most consistent starting point. It is claimed that a more consistent starting point would be to assume that there are no restrictions on political coalitions. I then investigate the consequences of this assumption.

The political-economy of distribution is studied for the case where there are no restrictions on coalitions by assuming that individuals can produce and they can also vote over distribution and that a winning coali- 
tion of voters can distribute resources in any way it wishes. This model is meant to capture the basics of a joint economic and political process in which the notion of democratic choice is taken seriously in as simple a manner as possible. The competition over distribution in this stripped down setting generates a number of questions. Among these are: would this competition always produce an unstable outcomes in the sense that there is no decision that cannot be defeated by a winning coalition? If not, under what conditions would a distribution be stable? How are outcomes affected by productivity? Would outcomes be unique? Would they be egalitarian? How does the power of wealth holders to prevent distribution affect the stability of the outcome? It should be noted of course, that stability is simply a characteristic of the system and does not carry any normative weight.

In order to answer some of these questions a condition of coalition stability drawn from cooperative game theory known as the core is used. To keep the paper focussed variations and extensions of this concept, and of the basic model, as well as empirical issues, will be dealt with in other papers.

All this, of course, needs some spelling out and there are some complexities in the question itself, as well as in the approach taken, to be discussed. To do this I survey the literature more fully in the next section.

The major results of the analysis are now briefly summarised as a prelude to the detailed development of the argument.

$[a]$. The value of individuals to society, understood in terms of their marginal productivity, is an important determinant of the outcome. Specifically, if the marginal contribution of every individual to production is constant, or increasing, there is a stable distribution that gives everyone as much, or more, than they can get by voting for change. In addition, this distribution always contains the egalitarian outcome.

$[b]$. The capacity of wealth holders to resist expropriation is also important. If individuals are not valuable in the pervious sense, but the marginal contribution of wealth to production is increasing at a sufficient rate, and wealth holders are able to prevent too much being expropriated, there is a distribution that cannot be challenged by any break-away coalition of voters.

$[c]$. If individuals are not valuable, and it is possible to expropriate most wealth, there is no stable distribution of the product.

$[d]$. Under some conditions there is a unique egalitarian outcome.

In cases where there is no stable distribution, and in some cases where there is, the final allocation may not be unique. This simply tells us that the outcome is not determined by the political power of coalitions but may depend on other factors. This should not be a surprise. All this paper tries to do is to make a start on the problem by determining what voting power buys in an unrestricted competition. I return to this point in the next section.

I set out the paper as follows. In section $\S .2$ the literature and the problem are discussed in more detail. In $\S .3$ the model is specified and it is analysed in $\S .4$. I study the egalitarian distribution in $\S .5$ for a more restricted production function and conclude in $\S .6$. For those less interested in the formal side of things I summarise the results of $\S .4$ and $\S .5$ at the beginning of those sections. The formal analysis that follows 
could be, largely, skipped.

\section{The literature and the interpretation of the problem.}

\subsection{The literature.}

The approach taken in this paper can best be understood by starting with one of the most common, although not of course the only, strands in the literature on the problem of political power and wealth distribution in liberal democracies. The common supposition has been that the poor, being in the majority would redistribute wealth without some check on their power. This supposition is found in eighteenth and nineteenth century debates about extension of the franchise to non-property holders. It occurs in the works of Bentham [7], and Madison [6], for example, and it continued to worry writers such as Schumpeter [24] and Hayek [9]. From a more recent perspective, Downs has also argued that competition for votes in a democracy would tend to transfer wealth from the rich to the poor $([5], 198)$. One response to the obvious lack of fit between this supposition and observed outcomes has been to ask, why don't the poor form a coalition that votes to take wealth from the rich? ([16], 9) or, as one writer puts it, 'why have the rabble not redistributed the wealth?' ([18], 359). This question has received considerable attention under the headings of: (i) media manipulation and dominant ideology [4], [12]; (ii) structural dependency [16]; (iii) wealth distortions of the political system [12]; and (iv) lack of information and incentive effects [5, 26]. In crude summary, the underlying problem that these works address is framed in the following manner. Since the poor don't use their votes to distribute wealth more evenly, something must be either distorting their preferences, or distorting the democratic process. In the dominant ideology approach the problem is exogenous preference distortion which makes the poor believe it is not in their interests, or is somehow unfair, to tax the wealth of the rich. In the structural dependency approach the political power of the majority is blocked by the ability of the wealthy to withhold investment, or to shift assets. The wealth distortion approach maintains that the rich exert influence on the political system through family connections and other forms of economic inducement. In arguments for incentive effects it is claimed that any attempted distribution will reduce the productivity of the rich and make everyone worse off. It could be claimed, for example, that some combination of these factors explains the frequent observation that, where voting is not compulsory, the poor turn out to vote at much lower rates than the rich. ${ }^{1}$

There is something to be said for each of these approaches and it seems reasonable to believe that each describes some of the factors that might be taken into account in a full description of the voting patterns of the poor. There are, however, some questions about whether they provide the appropriate starting point for an analysis of distribution. ${ }^{2}$

The problem is that these approaches start with the assumption that a coalition of the poor has priority over other possible coalitions in a conflict over distribution, and that the task to explain why this does not

\footnotetext{
${ }^{1}$ The poor see the democratic process as fairly ineffectual in terms of their main concerns, in other words. I owe this observation to the Chief Editor Public Choice.

${ }^{2}$ The alternative is to assume that distribution is only one dimension and can offset by other policy dimensions. See Roemer [21]. I wish to concentrate on distribution and do not pursue this line.
} 
form. Even overlooking the obvious contradiction, it is not clear why it should be assumed that this is the appropriate starting point. Why is a coalition of the poor any more natural than any other coalition of voters? ${ }^{3}$

It might be the case that there is a good answer to this question and that, under some conditions, the poor form a natural coalition, or are more homogeneous as a group than any other possible alliance. ${ }^{4}$ These conditions, or the assumptions that produce them, would need to be stated formally however, and built into the argument. ${ }^{5}$ Without such statement there is no good reason to begin by attempting to explain the absence of these coalitions and of a more egalitarian distribution under democratic procedures ([27], 390).

In Downs's work coalitions are restricted by the assumption that voters are linearly ordered. This still leaves essentially the same question. Why should coalitions be ordered in this way over distributional issues? ${ }^{6}$

An alternative approach to the problem would be to ask, what would happen if democracy were taken seriously as a winner take all competition without restrictions on coalitions of voters? What this does, of course, is to change the focus of the question from asking why a particular coalition does not form to asking, what outcomes would this competition produce?

The most straightforward way to deal with this question is to use a model in which parties, or candidates, offer a distribution in order to win power and are not constrained by any other considerations. ${ }^{7}$ Assume, for example, that a political entrepreneur starts by forming a coalition of the poor in order to transfer wealth from the rich. Maybe the entrepreneur stipulates a wealth cut-off point, or goes through the phone book and publicises the names of the people who will benefit. What stops a different entrepreneur buying out one or more members of the coalition of the poor to form a new winning coalition?

It has already been suggested by a number of writers, such as Tullock [26], Aumann and Kurz [1, 2], Putterman [18] and Ward [28], for example, that this type of unrestricted competition would be expected in democracy and often occurs, although sometimes in indirect forms. Tullock points out that, in real democracies, the rich and poor may sometimes even join the same coalition to vote over distribution. He also observes that, since the only thing that matters is power, 'there is no reason we should anticipate that the poor would do particularly well' $([26], 60)$ in this sort of coalition formation game. Aumann and Kurz point out that, if we aggregate tax rates and government spending, it is a simple matter to make payoffs to whatever groups make up a winning coalition. Putterman and Ward give a similar description of this

\footnotetext{
${ }^{3}$ In some ways the Marxian approach is more subtle in that it takes the conditions of the formation of the non-owners of the means of production as a group as a problem.

${ }^{4}$ The assumption that parties can only appeal to the poor or the rich could be justified indirectly by assuming a linear taxation schedule. See the recent papers by Przeworski, Peck and Roine, for example [17, 14, 22]. Why should distribution be restricted in this way?

${ }^{5}$ See Roemer, for example, for an attempt to endogenize wealth distribution in a game between a Tsar who proposes penalties and Lenin who proposes an income distribution [20].

${ }^{6}$ In this case, however, the distribution should not be egalitarian as he claims, but give everything to the median voter. Tullock [26] also makes this point.

${ }^{7}$ This view of competition is familiar from the work of Downs ([5], 28). It is supported by more recent studies on the increasing tendency of parties to buy support from fragmented groups of voters [3]. For criticisms and references to the literature see ([13], $278-80)$.
} 
competition. In addition they point out that it may produce endless cycling. ${ }^{8}$

It might be thought that the prospect of cycling might cause parties or candidates to enter into, as Putterman puts it, some sort of Pandora's box agreement not to become involved in an endless struggle. In this case the type of unrestricted competition I have described would not occur and, perhaps, it would have less analytical interest, As he points out, however, this is illogical, and does not justify the decision in much of the literature to ignore it [18]. ${ }^{9}$ This is, of course, correct. He does not attempt any further analysis, however.

In what follows I attempt to analyse the consequences of thus type of unrestricted competition over distribution in a system in which production and collective choice takes place simultaneously.

\subsection{The approach to be used.}

The natural techniques for analysing a competition between coalitions of voters over distribution are those of cooperative game theory. There has already been some sophisticated work in this field. The papers by Aumann and Kurz $[1,2]$ have a similar starting point to this paper in that they want to understand what would happen in a basic winner take all democracy. They study a model with continuum of voters in which there is some fixed amount of wealth and no production. Any possible coalitions can form, and the majority can impose any tax and redistribution it wishes. In response the minority can destroy all its endowments. In [1] they show that this would produce a marginal rate of tax between fifty and one hundred percent. This model is extended by Peck [14] to look at the case where there is a linear production function and a linear tax rate. In this model taxes effect the labour supply.

In common with Aumann and Kurz this paper retains the notions that any coalitions can form and that the losers can destroy resources. In other respects my paper follows a different line of attack in order to get more insight into questions of political power and stability and it also allows production. ${ }^{10}$ This is not to suggest that my approach is in any way better than theirs, or supersedes it. I am simply asking different questions.

From the perspective of questions about political power and stability, Aumann and Kurz solve the problem in terms of a 'reasonable compromise' ([1], 1142) between the claims of all individuals. ${ }^{11}$ In contrast, my paper is concerned with the set of offers that give each individual at least as much as their power and wealth gives them in any possible coalition. It represents what they can get for themselves. This is not the same as a fair share of the surplus. It is the existence of a distribution that satisfies these claims, and cannot be improved upon by any break away coalition of voters, that provides the idea of stability. As will be shown below, it is possible for a compromise solution to exist, such as the Shapley value, that does not meet this

\footnotetext{
${ }^{8}$ Unlike Putterman and Ward, Tullock argues that it might be possible to stabilize this by logrolling ([26], 60). This might be true, but, like $[5,28,18,1,2]$, this paper is concerned with understanding the basics of democratic competition and considers institutions a second order problem.

${ }^{9}$ Such an agreement is, obviously, not a Nash equilibrium.

${ }^{10}$ Aumann and Kurz say that production should be included, but do not do so ([1], 1137).

${ }^{11}$ More precisely, Aumann and Kurz use the Harsanyi-Shapley-Nash value in [1] and [2].
} 
stability condition.

In order to sharpen the distinction between this paper and other literature in the field there are some other approaches which might be mentioned. It would also be possible to interpret the competition in terms of a conflict over taxation as is done in a paper by Roine [22], for example. This differs from most of the literature in that it studies tax avoidance, rather than focussing on incentives, and avoidance could be seen as analogous to withholding assets. This offers valuable insights but, unlike my paper, it assumes a fixed tax rate rather than a pure democracy where the winner can take all. It is never clear in this paper, or in Peck's [14], why a competition over distribution should be limited to a particular tax schedule.

It is also possible to interpret the well known literature on rent seeking in terms of coalitions that attempt to extract resources from others. ${ }^{12}$ The difference is that rent seeking is commonly concerned with optimizing behaviour within an existing set of institutions. It could in principle, but usually does not, deal with the basics of a democratic competition where the winners get access to all resources. ${ }^{13}$

In this paper the theory of the core will be used to determine which distributions are stable and to study their properties. There are other related solution concepts that might be used to this end, such as the von-Neumann Morgenstern stable set, and the bargaining set. Each has particular merits, but the core is the most well known and straightforward, and this might be claimed to give it some additional application. It is also the most analytically tractable. ${ }^{14}$

It would, of course, be more satisfactory if the assumptions about the value of coalitions that are needed to use the theory of the core could be generated endogenously from an explicitly modelled bargaining process. A great deal of work has been done in this regard and more recent attempts, for example that of Ray and Vohra [19], have been made to generate payoffs and the coalition structure simultaneously. As it stands, however, this presents complex theoretical problems and any such work is best left for other papers. The first task is to get some insight into the competition over distribution for the simpler case.

For readers not familiar with the idea of the core, or who want some intuition about the ideas underlying the formal arguments, the analysis that follows might be thought of, roughly, as follows.

Imagine that a political entrepreneur wants to put together a winning coalition that can demand more for its members than an existing distribution. Since the value of this objection depends on what it can take away from the losing coalition, the losers will want to prevent the winners getting at their wealth. It follows that a credible claim by any coalition will depend on its productive capacity and its capacity to expropriate, given the reactions of the losers. What we need is a model that takes economic power, production and political power into account. In studying the core we are looking for the distributions against which no

\footnotetext{
${ }^{12}$ The literature on rent seeking is enormous although [25] is usually cited as the classic. See also ([13], 333-58).

${ }^{13}$ There is also a large literature on conflict over resources. See for example [10]. This is of less direct relevance because it deals with fighting between groups rather than competition in democracies. See [8] for the relation between this and the rent seeking literature.

${ }^{14} \mathrm{~A}$ disadvantage of the bargaining set for current purposes is that it is never empty.
} 
possible coalition can object when these various sources of power are dealt with simultaneously.

In some cases I will move away from unrestricted coalitions to look at the situation where the number of members of the coalition is fixed by the voting rule. This restriction is justified on grounds of interest. It might occur where the entrepreneur only has coarse information and wishes to limit offers to minimal winning coalitions.

\section{The model of competition over distribution.}

\subsection{The model.}

The social system is modelled as a democracy in which there is an arbitrarily large, but finite, number of individuals, $n$, that each begins with a specified holding of wealth which is used in production. Collective choices are made by voting and any coalition of voters of size $m \geq z>\frac{n}{2}$, where $z$ is specified by the choice rules, is winning. The set of feasible coalitions is written $\Omega$ and the all player coalition is written $N$. Coalitions are either winning or losing. The winning coalition is allowed to distribute the total product in any way it wishes, but the winners cannot enslave the losers. The losing coalition can destroy some of its initial endowment in order to deny its fruits to the winning coalition. As an alternative to destruction it is also possible that wealth could be shifted to some other political jurisdiction, as in the structural dependency literature [16]. It is assumed that either this is not possible, or the winning coalition could block receipts of revenue to equate any such move with hiding or destroying. ${ }^{15}$

The initial amount of wealth held by individual $i$ is $w_{i}$ for $i:=1, \ldots n$ and the total wealth is $\sum_{i=1}^{n} w_{i}:=\bar{w}$. In order to simplify the analysis wealth is treated as an input to production and all wealth is invested. It is also assumed that there is a bijective mapping between the amount of wealth held and the amount of social product that can be claimed, say $\psi: w_{i} \mapsto a_{i}$ where $w_{i}$ is the wealth held by $i$ and $a_{i}$ is the amount of the product. This means that distribution of the product and distribution of wealth are essentially the same. It might be thought that treating wealth as a factor of production misses something essential in the concern with unequal distribution. In this case the concern would be with wealth in disposal, or as giving access to power or status. This could be analysed using the approach developed in this paper, although the model may be simpler than that developed below.

It is also assumed that the produced good is measured in utilities and individuals prefer more to less. This means that the share of the product obtainable is transferable between the members of a coalition.

The value for a coalition is the maximum amount of the total product it can get for its members, taking into account the actions of its complement, written $S^{c}$. This is given by the characteristic function

$$
v: S \rightarrow \mathbb{R}
$$

\footnotetext{
${ }^{15}$ It would be possible to extend the framework to deal with these complications.
} 
and the game can be referred to as $v$.

The fraction of the wealth that a winning coalition can expropriate is written $b$ for some $0 \leq b \leq 1$. This means that $1-b$ can be thought of as the index of the capacity of initial holders to destroy wealth, or as the cost or shifting control of wealth from one group to another such as loss of production or any transaction costs. In a Marxian model $b$ would represent the power of labour against capital. In structural dependency theory $1-b$ could be rescaled to serve as an index of the capacity to shift capital.

It is also possible that the productivity of coalitions may vary according to their membership. It might be the case, for example, that a coalition $S$ can only obtain its maximum value if it contains a subset made up of certain individuals. This might be because these individuals are required to work the technology of production or to ensure support for the winning coalition. The details will, of course, depend on the particular context. ${ }^{16}$ The ability of a coalition to produce is called its effectiveness.

The simplest way to capture this idea is to say a coalition is fully effective when it contains some, or all, members from a set $K$ where $|K|<z$. When $K=\phi$ all coalitions are fully effective. When $K \neq \phi$ some coalitions are ineffective. Let $\bar{K}$ be $K$ partitioned into the subsets that contain sufficient individuals to make a coalition fully effective and let $\kappa_{i} \in \bar{K}$.

The characteristic function for a winning coalition with $|S|=m_{S} \geq z$ is

$$
v(S):=f\left(\hat{m}_{S}, c w_{S}\right)
$$

where $f$ is the production function, $\hat{m}_{S}$ the number of workers, and

$$
w_{S}:=\sum_{i \in S} w_{i}+b \sum_{j \in S^{c}} w_{j}
$$

with $c=1$ if a coalition is fully effective and $0 \leq c<1$ otherwise.

In order to specify the payoff for a losing coalition it is assumed that the best threat it can make is not to cooperate in the production process. In this case $\hat{m}_{S}=m_{S}$. For simplicity it is assumed that the payoff is the same for every losing coalition. Rescaling gives

$$
v\left(S^{c}\right):=f\left(n-m_{S}, 0\right)=0
$$

Although not necessary for the analysis, it simplifies matters to assume that each individual has equal voting weight. It is also assumed, for simplicity, that $n$ is sufficiently large that $f$ can be approximated by a continuously differentiable function.

\subsection{The core.}

The core is the set of distributions with the property that no coalition of players could get more for its

\footnotetext{
${ }^{16}$ Maybe the initial holders have some scarce talent that gave them the wealth in the first place and its value in production is reduced without their contribution. An equally plausible assumption is that talent can be reproduced.
} 
members by proposing an alternative feasible distribution and a distribution in the core is a stable outcome. ${ }^{17}$ The core is written $C(v)$. In a transferable utility game the conditions required for $C(v)$ to be non-empty are that there exists a vector $a:=\left(a_{1}, \ldots, a_{n}\right)$, such that

$$
\sum_{i \in S} a_{i} \geq v(S) \text { for all } S
$$

and

$$
\sum_{i \in N} a_{i} \leq v(N)
$$

for all $S \subset N$. A vector $a$ that satisfies these conditions with equality in condition (2) is called an imputation. since there is no reason for not satisfying equality we are only concerned with imputations. Note that the conditions required for the core lose their structure for the unanimity game where $z=n$ because only one coalition has a payoff. In this case the conditions are trivially satisfied by any $a=\left\{a: \sum_{i \in N} a_{i}=v(N)\right\}$. It might be argued that the egalitarian imputation is a better representation of the power of the players in this case. We are only concerned with $z<m$.

When the core is non-empty the set of imputations $C$ can be understood as the $n-1$ dimensional manifold in $\mathbb{R}^{n}$ specified by conditions (1) and (2). This has a volume in $\mathbb{R}^{n-1}$ given by the Lebesque outer measure $\mu: C \rightarrow \mathbb{R} .^{18}$ Reference to the core shrinking or expanding refers to the value of $\mu(C)$.

It will be noted that the directional derivative

$$
d \mu=\lim _{\epsilon \rightarrow 0} \frac{\mu\left(C(v)+\epsilon v\left(S_{i}\right)\right)-\mu C(v)}{\epsilon}
$$

exists for $C \geq 0$ and hence $\mu$ is differentiable in $v$ with $\frac{d \mu}{d v}>0$.

In order to study the properties of the core it is assumed, to begin with, that $f$ is homogenous of degree $\sigma$. In order to get sharper results, particularly in relation to the egalitarian distribution, I will then consider the special case where the production function is Cobb-Douglas.

\section{The core for the distribution game: $f$ homogenous of degree $\sigma$.}

\subsection{The main results.}

The main result is that the game always has a non-empty core when the marginal productivity of labour is constant or increasing. This is the first part of Theorem 2. One way to interpret this is to say that each member of society must be considered no less valuable than other members, or that each new member has at least as much to add to production as any existing member. The second part of Theorem 2 covers the case where the marginal productivity of labour is decreasing. In this case the core is only non-empty when

\footnotetext{
${ }^{17}$ In order to avoid a common mistake, it must be stressed that the core is an equilibrium in that no coalition has an incentive to deviate. It does not depend on a binding agreement.

${ }^{18}$ Although not in $\mathbb{R}^{n}$ of course since the Lebesque measure is zero.
} 
$\sigma \geq 1$ and wealth holders can prevent too much being expropriated. To get some rough intuition on this, imagine that all wealth can be expropriated and $b=1$. In this case every sub-coalition would be able to produce a large amount relative to the coalition of the whole and their claims could not be satisfied under any division of the total product. If, on the other hand, $\sigma \geq 1$, the core is empty even when all wealth can be destroyed and $b=0$. This is because the coalition of the whole can't produce enough to meet all claims.

Some of these results may seem counter-intuitive. It might seem surprising to say that, if it is too easy to expropriate wealth and $b$ becomes large, the poor may be unable to use their voting power to make an enforceable claim. This mystery disappears if we stop thinking about the poor as a natural coalition or in terms of compromise. What we are being told is that, if power is all that matters, no coalition can make an enforceable claim. ${ }^{19}$

It might also seem counter-intuitive to say that, if all wealth can be destroyed and $b=0$, the core may be empty since it might be thought that, if no-one has power to expropriate, there would be no alteration in the original distribution. The reason is that every coalition can make a claim on the total product as great as its stand alone claim and there is insufficient productivity to satisfy all claims.

It would also be interesting to know how an increase in the size of the coalitions required to win an election affects the core. ${ }^{20}$ This is a difficult question to answer since we cannot generate the size of coalitions endogenously. Although our intuition might be that coalitions are minimal winning this is not correct for a system with production. For some winning coalition, $S$, the value of an additional member when $b<1$ will depend on the production function and the value of $b$ and this may, or may not, offset losses to each existing member of $S$.

In order to deal with this the investigation is restricted to the case where coalitions are constrained to be minimal winning and $m=z$. It turns out that the volume of the core shrinks as $z$ increases for $n$ sufficiently large. This may be the result of the fact that, for a larger $z$, there are more people to satisfy in a winning coalition, and hence a smaller range of imputations that are acceptable.

Before establishing these results I show that, if the game where every coalition is fully effective has a non-empty core, then all games have a non-empty core. This tells us that reducing effectiveness increases rather than decreases the stability of the game.

\subsection{Existence.}

The existence of the core can be established directly for the case where the marginal productivity of labour is constant or increasing by showing that the game is convex. In other cases we need to use the BondarevaShapley theorem. In order to state this theorem we need the following definition.

\footnotetext{
${ }^{19}$ This illustrates the difference between Aumann and Kurz's approach and the approach adopted here. Using their solution concept, if $b=1$ the outcome would be completely egalitarian ([1], 1139).

${ }^{20} \mathrm{I}$ owe the suggestion to study the relation between $m$ and the core to an anonymous reviewer for Public Choice.
} 
Definition. Suppose $B$ is a finite subfamily of $\Omega$. Define $B$ as a balanced collection if for every $S \in B$ there exists an indexed set $y_{S}$ and $\sum_{S \in B} y_{S} \delta_{i}:=1$ where $\delta_{i}:=1$ for $i \in S$ and $\delta_{i}:=0$ otherwise. $B$ is minimally balanced if there is no $B^{\prime} \subset B: B^{\prime}$ is balanced.

Write $\gamma:=\sum_{S \in B} y_{S} v(S)$ and

$$
\varphi:=v(N)-\gamma
$$

Theorem 1 (Bondareva-Shapley) The core of the game $v: \Omega \rightarrow R$ is non-empty if and only if

$$
\varphi \geq 0
$$

for every minimally balanced collection.

For proofs see Ichiisi $([11], 81)$, and Peleg ([15], 40). I refer to a minimally balanced set as balanced in what follows. It is obvious that we only need to consider $\max \gamma$ in what follows.

In order to consider the impact of effectiveness on the existence of the core write the game with $K=\phi$ as $v$ and the game with ineffective coalitions as $v$.

Corollary 1 of Theorem 1. $C(\hat{v}) \neq \phi$ if $C(v) \neq \phi$.

Proof. For the balanced set of coalitions we have $v<v$ when at least one $\kappa_{i} \in \bar{K}$ is not contained in $S \cap K$ and $\dot{v}=v$ otherwise. It follows that $\dot{\gamma}<\gamma$.

What this says is that the result of reducing the effectiveness of some coalition, $S$, is that the payoff required to satisfy the claims of its members is reduced. It is assumed all coalitions are fully effective in what follows.

It is possible to establish some properties of the relation between $\mu$ and $\varphi$ from the definition of the set $B$ and Theorem 1 that are useful for the subsequent arguments.

Corollary 2 of Theorem 1. $[a]$. The core has measure $\mu>0$ for $\varphi>0$ and $\varphi$ and $\mu$ decrease as $v$ increases;

$[b] . \varphi$ is continuous in $v$.

Proof. [a]. Suppose that $f$ increases to give $v^{\prime}>v$ and the configuration of coalitions alters so that $\gamma_{B^{\prime}}\left(v^{\prime}\right) \leq \gamma_{B}(v)$. In this case $\gamma_{B^{\prime}}\left(v^{\prime}\right)$ cannot be the maximum value for $\gamma$ since, for the balanced set $B$, we have $\gamma_{B}\left(v^{\prime}\right)>\gamma_{B}(v) \geq \gamma_{B^{\prime}}\left(v^{\prime}\right)$. Therefore $\varphi$ must decrease.

Assume $\mu(v)>0$ and $\varphi(v)=0$. If $v$ increases, $\varphi$ decreases and $C$ is empty. Contradiction. Hence $\varphi>0$. In the other direction assume $\mu(v)=0$ and $\varphi(v)>0$. If $v$ increases by any amount $\epsilon>0$ we have $C$ becomes 
empty. Hence $\varphi(v+\epsilon)<0$ and $\varphi$ must be discontinuous at $v$ because the balanced set that gives $\max \gamma$ switches from $B$ to $B^{\prime}$. This means that $\lim _{\epsilon \rightarrow 0} \gamma_{B^{\prime}}>\gamma_{B}$. Since $B^{\prime}$ exists at $v$ and $v+\epsilon$ and $\gamma$ is continuous for a given $B$ it must be the case that $\lim _{\epsilon \rightarrow 0} \gamma_{B^{\prime}}-\gamma_{B}<\delta$ for some $\delta>0$ and hence $\varphi$ is continuous. By the intermediate value theorem it must pass through $\varphi=0$. It follows that at this is the value which gives $\max \gamma$ at $\mu=0$. This contradicts the original assumption.

$[b]$. This follows immediately from part $[a]$.

In other words, $\varphi$ and $\mu$ move in the same direction. It is obvious that, for $\varphi=0$, we have $\mu=0$ and that $\frac{\partial v}{\partial b}>0$.

This allows us to consider the conditions for the core to be non-empty.

Theorem 2 . The core of the game $v$ is non-empty for all initial distributions for:

$[a]$. all $b$ when the marginal productivity of labour constant or increasing;

$[b]$. all $b \in[0, s]$ for some $s<1$ for $\sigma \geq 1$ when when the marginal productivity of labour is decreasing.

Proof of Theorem. $[a]$. Suppose $b=1$ and the marginal productivity of labour constant or increasing. The equivalent condition to that stated above for convexity is that $v(S \cup i)-v(S) \geq v(S)-v(S-i)$. This is obviously true for $b=1$ since $w_{S}=\bar{w}$ for all $S$. From Corollary 2 of Theorem 1 it is true for all $b$.

$[b]$. The idea is to show that the core of the game is non-empty for $\sigma=1$ if and only if $b=0$ and then to show that it expands as $\sigma$ increases.

If. For $\sigma=1$ and $b=0$ each coalition of size $m_{S}$ has its initial distribution $\sum_{i \in S} w_{i}=w_{S}$. It follows that

$$
\gamma=\sum_{S \in B} y_{S} f\left(m_{S}, w_{s}\right)=f\left(\sum_{S \in B} y_{S} m_{S}, \sum_{S \in B} y_{S} w_{s}\right)=f(n, \bar{w})
$$

and hence $\varphi=0$.

Only if. Assume $\sigma=1$ and $b>0$. From the previous $\gamma>f(n, \bar{w})$ which gives the contradiction.

Consider $\sigma>1$. For $\sigma=1$ we have from the previous

$$
\sum_{S \in B} y_{S}\left(\frac{w_{s}}{\bar{w}}\right)^{\sigma} f\left(\frac{m_{S}}{w_{s}}, 1\right)=f\left(\frac{n}{\bar{w}}, 1\right)
$$

for any balanced set. Since $\left(\frac{w_{s}}{\bar{w}}\right)^{\sigma}$ decreases as $\sigma$ increases it must be he case that $\varphi$ is increasing and hence $\varphi>0$ for $\sigma>1$.

Now consider $b>0$. Since $\varphi>0$ and hence $\mu>0$ at $b=0$ and $\mu$ is continuously differentiable in $b$ there must be some $s>0: \mu \geq 0$ for $b \in[0, s)$.

It follows immediately from the proof of part $[b]$ of Theorem 2 and the fact that $\mu$ is differentiable in $v$ that $\frac{\partial \mu}{\sigma}>0$.

Corollary 1 of Theorem 2. $\mu$ decreases as $z$ increases for $n$ sufficiently large. 
Proof. Set $m=z$. it is easy to show that the total number of coalitions in a balanced set is $n$ and that $y_{S}=\frac{1}{m}$. From the proof of part $[b]$ we have

$$
\gamma=\sum_{S \in B} m^{\sigma-1} f\left(1, \frac{w_{S}}{m}\right)
$$

By choosing $n$ sufficiently large we can approximate $\gamma$ with a differentiable function and make $\frac{\partial \frac{w_{S}}{m}}{m}$ as small as we like. It is immediate that $\frac{\partial \gamma}{\partial m}>0$ and hence $\varphi$ decreases as $m$ increases.

In order to get some more insights I now concentrate on a more restricted set of production functions.

\section{Properties of the core: $f$ Cobb-Douglas.}

\subsection{The main results.}

The properties of the core are now studied in more detail for the special case where the production function is Cobb-Douglas. This has the advantage that it allows us to talk about the marginal productivity of labour and of capital separately and to investigate their affect on distribution. The production function is now written

$$
f_{S}:=m_{S}^{\alpha}\left(w_{S}\right)^{\beta}
$$

where $\alpha, \beta>0$ and $\alpha+\beta=\sigma$. It follows immediately from the fact that $\frac{\partial \mu}{\sigma}>0$ that the volume of the core increases as $\alpha$ or $\beta$ increase. It is assumed in everything that follows that $\sigma \geq 1$ to avoid trivial cases.

The most important result is given in Theorem 3. It says that, if the marginal productivity of labour is increasing, the core always contains the egalitarian distribution. This does not necessarily mean, of course, that this is the final distribution. It also shows that, when the marginal productivity of labour is decreasing, the necessary condition for the core to contain the egalitarian distribution is that the initial distribution is not too unequal, and the proportion of the total wealth that can be claimed by any winning coalition is not too large. This more or less says that coalitions must be able to take enough to even things up. If they can take too much the only imputations in the core are non-egalitarian.

It might be expected, for the case where the constraint $m=z$ is placed on coalitions, that an increase in the number of people that have to agree would tend to bring the egalitarian distribution into the core. The intuition would be that if something close to all individuals have to agree everyone has roughly the same power. This guess turns out to be wrong. If the egalitarian distribution is not in the core an increase in $z$ will not cause it to be included. If it is in the core an increase in $z$ may cause it to drop out.

From the other side it might be asked, when is the egalitarian distribution not in the core? This will occur when the initial distribution is sufficiently unequal and the capacity to expropriate wealth is sufficiently low. The intuition is that initial distributions matter and the coalition of the rich can always claim enough under 
these conditions to block an egalitarian distribution.

This leaves the question, under which conditions is the egalitarian distribution unique? In the corollary it is shown that this will be the case when all wealth can be expropriated and the marginal productivity of labour is constant. The requirement that all wealth can be expropriated for the egalitarian distribution to be unique is intuitively plausible. The reason $\alpha=1$ is needed is, from part $[a]$ of Theorem 2 , that if $\alpha>1$ the core will contain more points than the egalitarian outcome and if $\alpha<1$ the core will cease to exist if $b=1$. It might seem a surprise that the value of $\beta$ is irrelevant for the uniqueness of the egalitarian outcome. The explanation is that, since every coalition has access to all wealth, a change in productivity will alter the amount available but will not alter the way in which it is distributed.

It will be noted that, in Aumann and Kurz's paper [1], there is an egalitarian distribution whenever all resources can be expropriated $([1], 1139)$. This is because, in their paper, every coalition has the same bargaining power and the value solution requires an equal division. In this paper, expropriation of all resources is not enough to produce the egalitarian solution. In addition the core will be empty when $b=1$ when $\alpha<1$ even though the fair division still exists.

In order to get some feel for these results, and for the way in which the size of the core changes for changes in $\alpha$ and $b$ note, from Theorem 2 part $[b]$, that for each $\alpha<1$, there is a $b$ sufficiently small such that $\varphi>0$ and a $b$ sufficiently large such that $\varphi<0$. From the intermediate value theorem this means that, for $\alpha<1$, there is a $b$ such that $\varphi=0$ and $C(v)$ is contracted to a single point. See fig. 1 for an example.

In this light what the discussion boils down to is, what is the relation between $\alpha$ and $b$ and the egalitarian distribution?

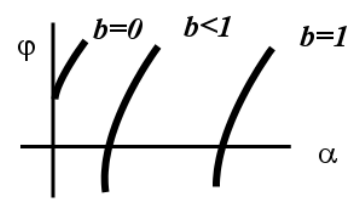

Figure 1. Example of the relation between $\alpha, b$ and $\varphi$ for $\beta=1$.

\subsection{The egalitarian distribution.}

The results for the egalitarian distribution are given in the following theorem.

Theorem 3 . The egalitarian distribution is in the core:

$[a]$. for all initial distribution and all $b$ and $\beta$ when $\alpha \geq 1$;

[b]. if and only if $\frac{\bar{w}}{\max w_{S}}>k$ for some $k$ sufficiently large when $\alpha<1$. 
Proof. [a]. The egalitarian distribution is $e=\left(e_{1}, \ldots, e_{n}\right)$ where $e_{i}=n^{\alpha-1} \bar{w}^{\beta}$ for all $i$ and $\sum_{S} e_{i}=$ $m_{S} n^{\alpha-1} \bar{w}^{\beta}$. Suppose $b=1$. In this case we have $v(S)=m_{S}^{\alpha} \bar{w}^{\beta}$ and hence $\sum_{S} e_{i} \geq v(S)$ as required by condition (1) for the core and condition (2) follows from the properties of $e$. Since $\frac{\partial v(S)}{\partial b}>0$ the proof is complete.

[b]. Only if. Suppose $e \in C(v)$. Then from the properties of $e$ we must have $\frac{\bar{w}}{\max w_{S}} \geq\left(\frac{n}{m_{S}}\right)^{\frac{1-\alpha}{\beta}}$ in order to meet condition (1).

If. Let $\frac{\bar{w}}{\max w_{S}} \leq\left(\frac{n}{m_{S}}\right)^{\frac{1-\alpha}{\beta}}$. Then condition (1) is violated.

Part $[a]$ gives the connection between increasing marginal productivity and an egalitarian imputation, and part $[b]$ shows that this is in the core if the initial distribution is sufficiently egalitarian and $b$ is sufficiently low. In order for part $[b]$ to be interesting it must be the case that a $\frac{\bar{w}}{\max w_{S}}$ exists such that the core contains the egalitarian distribution. Observe that this term takes its maximum value of $\frac{n}{m}$ for the egalitarian initial distribution $\frac{m \bar{w}}{n}$ and $b=0$. In this case $\frac{n}{m}>\left(\frac{n}{m}\right)^{\frac{1-\alpha}{\beta}}$ and hence $e \in C(v)$. It follows from the inequality sign that $k: e \in C(v)$ for some $b \in[0, s)$ and for some $\frac{\bar{w}}{\max w_{S}}<\frac{m \bar{w}}{n}$.

In order to investigate the relation between the value of $z$, for the case where the constraint $m=z$ is in force, consider again the condition $e \in C(v)$ requires $\frac{\bar{w}}{\max w_{S}} \geq\left(\frac{n}{m}\right)^{\frac{1-\alpha}{\beta}}$. Apart from the previous speculation about agreement in larger coalitions, it might also be guessed that an increase in $z$ will reduce the right hand side and make the egalitarian imputation more likely. What happens is this.

Corollary 1 of Theorem 3. Suppose $\{m: m=z$ for all $z\}$ and that $e \notin C(v)$ for some $z$. Then $e \notin C(v)$ for all $z<z^{\prime}<n$.

Proof. Interpolate $\theta=\frac{\bar{w}}{\max w_{S}}$ and $\psi=\left(\frac{n}{m}\right)^{\frac{1-\alpha}{\beta}}$ for $m=z$ with continuous functions. The result is immediate from the first and second derivatives $\frac{\partial \theta}{m}<0, \frac{\partial^{2} \theta}{m^{2}}>0$ and $\frac{\partial \psi}{m}<0, \frac{\partial^{2} \psi}{m^{2}}<0$ and the fact that, even thought the core is not defined at $m=z=n$ we see $\theta \rightarrow 1$ and $\psi \rightarrow 1$ as $m \rightarrow n$.

It was also said that an increase in the minimal size of a winning coalition may shrink the core in a manner that forces an egalitarian distribution out of the core. This follows immediately from the first and second derivatives on $\theta$ and $\psi$ in the above proof.

In order to consider the conditions under which the egalitarian distribution is the only point in the core consider fig. 1. Although there exist single point imputations given for $\varphi=0$ we do not know whether any one of these gives the egalitarian distribution. Alternatively, there might be several points where $\varphi=0$ which correspond to the egalitarian imputation.

It is possible to throw some light on this problem by noting, from part $[a]$ of Theorem 3 , that, if the core can be contracted to a point for $\alpha \geq 1$, it must give the egalitarian distribution. This idea is behind the proof given below. See fig. 2 for an illustration. 
Corollary 2 of Theorem 3. The egalitarian distribution is the unique point in the core for all initial distributions if and only if $\alpha=1$ and $b=1$.

Proof. If. For $\alpha=1$ and $b=1$ we have $\sum_{S} e_{i}=m \bar{w}^{\beta}$ and hence $\varphi=n \bar{w}^{\beta}-n \bar{w}^{\beta}$. This means $\varphi=0$ and $C(v)=e$.

Only if. Suppose $\varphi=0$ for some $b<1$. Then we need $n^{\alpha} \bar{w}^{\beta}=\frac{n}{m} m^{\alpha} \tilde{w}^{\beta}$ where $\tilde{w}$ is the average holding and hence $\tilde{w}<\bar{w}$. This requires $n^{\alpha-1}<m^{\alpha-1}$ and hence $\alpha<1$. Since $e \in C(v)$ for all initial distributions only if $\alpha \geq 1$ this establishes the contradiction. Hence $b=1$. In this case $\varphi=0$ requires $\alpha=1$.

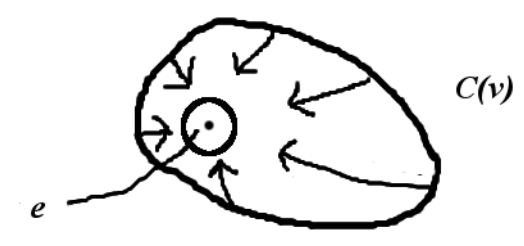

Figure 2. Contraction of the core.

\section{Conclusion.}

The aim of this paper was to analyse the political-economy of distribution by constructing a simple model made up of a production function and a democratic choice procedure in which the winning coalition determined the distribution of output. The theory of the core was used to get insights into the outcomes that coalitions can guarantee themselves as a result of their political and economic power. Unlike approaches which use a value solution, the core does not, usually, determine a unique outcome. It is an open question whether, in the absence of stability or a unique outcome in the core, the system would converge to the outcome given by a value solution. Maybe ideology, and the factors mentioned in the discussion of the literature in $\S .2$, have a part to play in these cases. It is only possible to discuss these questions in a rigorous way, however, if we have the sorts of results produced in this analysis as a guide.

The rest of this conclusion is used to speculate a little on what some of the results might be hinting at. Since this is only a first slice at the problem it is dangerous to extrapolate too far, but some of the results generate interesting reflections. It was found, for example, that where every individual is valuable the core is always non-empty and contains the egalitarian distribution. Is it generally the case that individuals need to be valuable economically, as well as to have voting rights, to create a stable, or even a more equal, society? There is some possible connection here with Przeworski's (2005) claim that democracies are stable if they have a sufficiently high per-capita income, although the relation between productivity and income would need to be established. ${ }^{21}$ In addition it was shown that if it is too easy to expropriate wealth there may

\footnotetext{
${ }^{21}$ I owe this point to a reviewer for Public Choice.
} 
not be any stable distribution. What do we read into this? If the costs of transferring wealth are very low might the result be turmoil or an abrogation of democracy? It would be interesting to scour the historical literature with these questions in mind. It is plausible that they might both be given a positive answer, but no claims can be made without a proper study.

It is not so clear what the finding that increasing the size of a minimal winning coalition may cause the outcome to be less egalitarian suggests. It is difficult to interpret it simply in terms of voting rules because the model includes the power derived from wealth and I leave it without further speculation.

It will be noted that, apart from a brief mention in $\S .2$, nothing has been said about incentive effects, as opposed to losses in transferring wealth. This seems to surprise some economists. Although it would be possible to take these into account, I agree with Aumann and Kurz and Roine [1, 2, 22], for example, that the impact of these has probably been overrated. Their inclusion would not add much of interest.

I think that a more interesting extension of the model would be to look for the kernel or nucleolus of the game, although this presents theoretical problems. It might also be possible to increase the dimensions of the model by allowing for a second, non-produced, good such as nationalism. It would be interesting to see how robust the results are under these extensions and whether they can be refined. 


\section{Acknowledgements.}

This paper has been much improved by the suggestions of two anonymous reviewers for, and the Chief Editor of, Public Choice. I benefited greatly from discussions in seminars in the Department of Economics, University of Massachusetts, the Department of Economics and Econometrics, University of Regensburg and the Department of Political Science, University of Calgary. I wrote the paper while the Helen Sheridan Chair of Economics at the University of Massachusetts.

\section{References}

[1] Aumann R. and M. Kurz. 1977 'Power and Taxes.' Econometrica vol. 45. 1137-1161.

[2] Aumann, R. and Kurz. 1978. 'Power and taxes in a multi-commodity economy.' Journal of Public Economics. vol. 9. 139-61.

[3] Dalton, R.J. 2000 The Decline of Part Identifications, in Dalton, R.J. and M. P. Wattenberg (eds). Parties without Partisans. Oxford, Oxford University Press,

[4] Gramsci, A. 1971. Prison Notebooks. International Publishers. New York.

[5] Downs, A. 1957. An Economic Theory of Democracy. Harper and Row. New York.

[6] Hamilton, A, J. Madison, J. Jay. 1961. The Federalist Papers. New American Library. New York.

[7] Gunn, J. 1988. 'Jeremy Benthamm and the Public Interest' in J, Lively. Modern Political Theory from Hobbes to Marx Key Debates. Routledge. London.

[8] Hausken, K. 2005. 'Production and conflict models versus rent seeking models.' Public Choice. Vol 123. $59-93$

[9] Hayek, F. 1973. Economic Freedom and Representative Government. London Institute for Policy Studies.

[10] Hirshleifer, J. 1995. 'Anarchy and its breakdown.' Journal of Political Economy. vol. 103. no.1. 27-52.

[11] Ichiishi, T. 1983. Game Theory for Economic Analysis. Academic Press. New York

[12] Miliband. R. 1969. The State in Capitalist Society. Basic Books. New York.

[13] Mueller, D. 2003. Public Choice III. Cambridge UniversityPress. Cambridge.

[14] Peck, R. 1986. 'Power and linear income taxes: an example.' Econometrica vol. 54. 87-94.

[15] Peleg, B. and P. Sudholter. 2003. Introduction to the Theory of Cooperative Games. Kluwer. Netherlands

[16] Przeworski, A. 1990. The State and the Economy under Capitalism. Harwood Academic Publishers. Chur. Switzerland.

[17] Przeworski, A. 2005 'Democracy as an equilibrium.' Public Choice. vol. 123. 253-73. 
[18] Putterman, L. 1996. 'Why have the rabble not redistributed wealth? in Roemer, J. (ed) Property Relations, Incentives and Welfare. St. Martin's Press. New York. 359-89

[19] Ray, D., and R. Vohra. 1999. 'A theory of endogenous coalition formation.' Games and Economic Behaviour. Vol. 26. 286-336.

[20] Roemer. J. 1985. 'Rationalizing revolutionary ideology.' Econometrica. vol.53. 85-108.

[21] Roemer. J. 1999. 'The democratic political economy of progressive income taxation.' Econometrica. vol.67. 1-19.

[22] Roine, J. 2006. 'The political economy of not paying taxes.' Public Choice. Vol. 126. 107-134.

[23] Shubick M., 1984 A Game-Theoretic Approach to the Political Economy, (Game Theory in the Social Sciences, Vol 2). MIT Press. Cambridge MA.

[24] Schumpeter. 1975. Capitalism Socialsim and Democracy. Harper. New York.

[25] Tullock, G. 1967. 'The welfare costs of tariffs, monopolies and theft.' Western Economic Journal. vol. 5. 224-32.

[26] Tullock, G. 1971. 'The charity of the uncharitable.' Western Economic Journal. vol. 9. 379-392.

[27] Wallerstein, M. 1996. 'Comment,' in Roemer, J. (ed) Property Relations, Incentives and Welfare. St. Martin's Press. New York. 390-393

[28] Ward. B. 1961. 'Majority rule and allocation.' The Journal of Conflict Resolution. Vol 5. 379-89. 